\title{
Literarische Rezeption und Intermedialität des Werks Der Vorleser: Anwendungen im Hörsaal Literaturübersetzen Deutsch- Spanisch
}

\section{(Literary reception and intermediality in Der Vorleser: applications to the course Literary Translation German-Spanish)}

\author{
María Pilar Castillo Bernal \\ z92cabep@uco.es \\ Grupo HUM-947: Textos, Ciencia y Traducción
}

Fecha de recepción: 14 de enero de 2014
Fecha de aceptación: 17 de febrero de 2014

Zusammenfassung: Ziel dieser Studie ist die Rezeption des Romans Der Vorleser von Bernhard Schlink bei den Studenten der Fakultät Übersetzen, sowohl als Leser als auch als Übersetzer des Werks. Als pädagogisches Hilfsmittel zur Übersetzung wird anhand der Verfilmung ebenso die Rolle der Intermedialität während des Unterrichts- und Lernprozesses untersucht. Hiermit sollen die Eigenschaften bestimmt werden, die die Rezeption des Werks seitens der Studenten beeinflussen und ein Vergleich mit einer anderen Leserschaft, den Schülern eines deutschen Gymnasiums, vorgenommen werden, um so einen Lehransatz zu erstellen, der als Übersetzungsrichtlinie der im Unterricht vorgestellten Absätze dient.

Schlüsselwörter: Rezeption, Lektüre, Intermedialität, Literaturübersetzen, Lehre.

Abstract: This paper focuses on the reception of the novel Der Vorleser by Bernhard Schlink by university students of translation, who are both readers and translators of the text. Furthermore, the use of the film adaptation as teaching aid and the role of intertermediality in the learning process will be examined. The purpose is to establish which factors have an impact on the reception of the novel by the students, in comparison to a different reader profile such as a German student of secondary education. The results are used to develop a teaching approach in accordance with the aims set in the curriculum of the course Literary Translation.

Key words: Reception, Reading, Intermediality, Literary Translation, Teaching. 


\section{DiE LITERARISCHE REZEPTION: DIE ROLLE DES LESERS IM WERK}

Seit den 60er Jahren ist die Rolle des Lesers im literarischen Werk Studienobjekt verschiedener Kritiker $^{1}$. Bis zu welchem Grad kann ein Kunstwerk an sich und speziell ein literarisches Werk als feste und unbewegliche Schöpfung gesehen werden? Und wie weit wird der Empfänger in diesen Werken mit einbezogen? Diese Themen wurden von Autoren wie Valéry oder Jorge Luis Borges ${ }^{2}$ angerissen und spiegeln sich in der Rezeptionstheorie- oder ästhetik wider.

Zweifellos ist der Gründer dieser Theorie Hans Robert Jauß, der 1967 seine Vorlesung an der Universität Konstanz unter dem Titel „Literaturgeschichte als Provokation der Literaturwissenschaft" hielt. Diese neue Literaturtheorie bricht mit dem historischen Objektivismus und den früheren Literaturtheorien, die das Werk als absolutes und geschlossenes System (Formalismus) oder als einfache Mimesis der sozialen Wirklichkeit (marxistischen Ästhetik) ansehen. ${ }^{3}$ Jauß besteht auf den Wiederaufbau des Erwartungshorizontes beim Leser, ein Konzept, das der Philosoph HansGeorg Gadamer aufnimmt, um die Rezeption des Werks und dessen Wirkung zu beschreiben. Tatsächlich besteht der Erwartungshorizont aus folgenden Faktoren: 1. vorhergehende Erfahrung des Lesers in dieser literarischen Gattung, so zum Beispiel, wenn der Leser bereits Werke des Autors oder derselben Literaturgattung gelesen hat, was der Fall wäre, wenn vor der Lektüre des Quijote bereits Romane über das Rittertum gelesen wurden; 2. die Form und Thematik der vorherigen Werke, die er bereits zur Kenntnis genommen hat; und 3. die Gegenüberstellung von poetischer und

1 Siehe Acosta, L.A., El lector y la obra: teoría de la recepción literaria (1989), Bennet, A. (Hg.), Readers and reading (1995), Freund, E., The return of the reader: reader-response criticism (1987), Holub, R.C., Reception Theory: A critical introduction (1989), Mayoral, J.A. (Hg.), Estética de la recepción (1987), Warning, R. (Hg.), Rezeptionsästhetik. Theorie und Praxis (1975), Iser, W., Der Implizite Leser - Kommunikationsformen des Romans von Bunyan bis Beckett (1972), Jauß, H.R., Kleine Apologie der ästhetischen Erfahrung (1972) y Eco, U., The Role of the Reader: Explorations in the Semiotics of Texts (1979).

${ }^{2}$ Diesbezüglich siehe Blüher (1986:458): "Muy pronto ya, en febrero de 1915, Valéry señala que «(siempre) que se habla de literatura hay que hablar necesariamente del lector» (C, V, p. 575). Veinte años más tarde, Valéry sigue todavía sorprendido de constatar que «nunca hay cuestión en los libros sobre literatura (de este) elemento esencial (que es) el lector» (C, XVIII, p. 411). Para Borges también es el lector y la lectura de las obras lo que cuenta: «El libro (...) es el diálogo que entabla con su lector (...). Una literatura difiere de otra, ulterior o anterior, menos por el texto que por la manera de ser leída» (PC, II, pp. 271-272)".

3 Jauß (1975:128): „Eine Erneuerung der Literaturgeschichte erfordert, die Vorurteile des historischen Objektivismus abzubauen und die traditionelle Produktions- und Darstellungsästhetik in einer Rezeptions- und Wirkungsästhetik zu fundieren. Die Geschichtlichkeit der Literatur beruht nicht auf einem post festum erstellten Zusammenhang literarischer Fakten, sondern auf der vorgängigen Erfahrung des literarischen Werkes durch seine Leser." 
praktischer Sprache ${ }^{4}$. Letzterer bedeutet, dass der Leser ein neues Werk sowohl ganz konkret im literarischen Erwartungshorizont, als auch im weitem Kontext seiner Lebenserfahrung begreifen kann:

\begin{abstract}
„Der dritte Faktor schließt ein, dass der Leser ein neues Werk sowohl im engeren Horizont seiner literarischen Erwartung als auch im weiteren Horizont seiner Lebenserfahrung wahrnehmen kann“ (Jauß, 1975: 132-133).
\end{abstract}

Eine wichtige Rolle spielt hier die sogenannte gesellschaftliche Funktion der Literatur, wonach die literarische Erfahrung zum einen die Weltanschauung des Lesers verändert und sich letztlich auf sein Sozialverhalten niederschlägt:

„Die gesellschaftliche Funktion der Literatur wird erst dort in ihrer genuinen Möglichkeit manifest, wo die literarische Erfahrung des Lesers in den Erwartungshorizont seiner Lebenspraxis eintritt, sein Weltverständnis präformiert und damit auch auf sein gesellschaftliches Verhalten zurückwirkt“" (Jauß, 1975: 148).

Gemäß Jauß’ These wird der Erwartungshorizont der Leserschaft, der aus den vorhin erwähnten Elementen besteht, eine Veränderung vollziehen, wenn ein Werk, aufgrund der Neuartigkeit beim Leser neue Erfahrungen verursacht.

Zusätzlich zu diesen von Jauß entwickelten Postulaten, die einen Paradigmenwechsel für die Literaturtheorie beinhalten, stellt Wolfgang Iser ${ }^{5}$ eine Theorie über den Lesevorgang ${ }^{6}$ oder "Akt des Lesens" auf. Iser unterscheidet beim literarischen Werk zwischen zwei Pole: das

${ }^{4}$ Jauß (1975:132): „Die Möglichkeit, den Erwartungshorizont zu objektivieren, ist aber auch bei historisch weniger profilierten Werken gegeben. Denn die spezifische Disposition für ein bestimmtes Werk, mit der ein Autor bei seinem Publikum rechnet, kann beim Fehlen expliziter Signale auch aus drei allgemein voraussetzbaren Faktoren gewonnen werden: erstens aus bekannten Normen oder der immanenten Poetik der Gattung, zweitens aus den impliziten Beziehungen zu bekannten Werken der literarhistorischen Umgebung und drittens aus dem Gegensatz von Fiktion und Wirklichkeit, poetischer und praktischer Funktion der Sprache, der für den reflektierenden Leser während der Lektüre als Möglichkeit des Vergleichs immer gegeben ist."

5 Siehe Enríquez Aranda, María Mercedes (2007:36): "La estética de la recepción (...) nace a finales de la década de los años sesenta en la entonces República Federal de Alemania y se personifica en un grupo de estudiosos localizados mayoritariamente en la Universidad de Constanza. Del año 1967 datan los que se consideran primeros manifiestos programáticos de esta nueva teoría: Für eine Literaturgeschichte des Lesens von Weinrich (1971) y Literaturgeschichte als Provokation der Literaturwissenschaft von Jauß (1971). De 1968 es también el discurso de Iser Die Apellstruktur der Texte, que cierra el trío de primeras incursiones en la nueva estética de la recepción".

${ }^{6}$ Iser, W., Der Lesevorgang, in Warning, R. (Hg.). 
Künstlerische, geschaffen vom Autor, und das Ästhetische, hervorgerufen vom Leser. Er behauptet, dass

„Das Werk ist das Konstituiertsein des Textes im Bewusstsein des Lesers." (Iser, 1975: 253).

Die Theorie der Unbestimmtheitsstellen von Roman Ingarden dient als Grundlage: Iser versteht die Lektüre als einen dynamischen Prozess, in dem der Leser anhand von schematisierten Ansichten (was gesagt wird) und den Leerstellen im Text (was nicht gesagt wird) die Bedeutung durch Formulierung und Berichtigung der Hypothese im Laufe der Lektüre erschließt. Dieser Prozess beinhaltet Antizipationen und Rückkoppelung seitens des Lesers, so dass es sich hier nicht um eine feste oder lineare Dynamik handelt. Anders ausgedrückt: der literarische Text ist nicht vollständig bis er nicht bewusst vom Leser gelesen wurde. Das heißt: nur wenn der Leser alle Leerstellen des Textes vervollständigt hat, hat dieser seinen Sinn und Zweck erreicht.

Die Tragweite dieses von Iser beschriebenen Prozesses ist folgende: Erstens stellt Iser direkt die Beteiligung des Lesers am Werk mit dem ästhetischen Genuss in Zusammenhang:

„Denn das Lesen wird erst dort zum Vergnügen, wo unsere Produktivität ins Spiel kommt, und das heißt, wo Texte eine Chance bieten, unsere Vermögen zu betätigen." (1975: 254).

In diesem Sinne haben die Leerstellen des Textes die Aufgabe den Leser in die Handlung einzuführen, inn zu beteiligen, da seine Kreativität angeregt wird. Zweitens wird argumentiert, dass bei der Produktivität des Lesers gewisse Toleranzgrenzen bestehen, so dass bei zu vielen Leerstellen Ermüdung auftritt oder bei einem zu offensichtlichen Text der Leser gelangweilt wird. ${ }^{7}$

\footnotetext{
${ }^{7}$ Iser (1993:10): "Texts with such minimal indeterminacy tend to be tedious, for it is only when the reader is given the chance to participate actively that he will regard the text, whose intention he himself has helped to compose, as real. For we generally tend to regard things that we have made ourselves as being real. And so it can be said that indeterminacy is the fundamental precondition for reader participation." In diesem Sinne behauptet Umberto Eco, dass "un texto, tal como aparece en la superficie (o manifestación) lingüística, representa una cadena de artificios expresivos que el destinatario debe actualizar" (1993:73). Eco hebt hervor, dass der Autor für den Leser die Aktualisierungsmechanismen im Text vorhersehen muss und eine textgemäße Strategie erschafft, auch wenn die Kenntnisse des Publikums nicht genau den Erwartungen des Autors entsprechen. Was die Interpretationsmöglichkeiten betrifft, ist bei Ecos Theorie die Unterscheidung zwischen "offenen" und "geschlossenen“ Texten interessant. So is ein "geschlossener" Text jener, bei dem der Autor einen Modell-Leser bestimmt und seine textgemäße Strategie präzise festlegen hat. Trotzdem ist es gemäß Eco aussreichend, dass ein
} 
Wie wir bereits gesehen haben, ist eine der Neuerungen, die diese Theorie einbringt, die aktive Rolle die dem Leser zugeteilt wird, auch wenn Kritiker argumentieren, dass hier sowohl die Untersuchungen über formale Aspekte und den kreativen Prozess des Werks aufgegeben werden ${ }^{8}$, als auch eine Aufbauanalyse dargestellt wird, besonders beim Roman der letzten Jahrhunderte ${ }^{9}$. Ohne diese Aufstellungen zu verallgemeinern, bietet uns die Rezeptionstheorie trotzdem äußerst wichtige Ansichten für den Hörsaal Übersetzen in Bezug auf das signifikante Lernen der Studenten, das nur durch Einbeziehung, Teilnahme und Motivation möglich ist, welche die grundlegende Voraussetzung zum Übersetzen ist, da auch der Übersetzer bewusst den Originaltext liest, ergänzt und danach in die Zielsprache neu ausarbeitet.

\section{DER ÜBERSETZUNGSSTUDENT ALS LESER DES LITERARISCHEN WERKS}

Aus Sicht der Übersetzung ist die Lektüre eines literarischen Werks eine komplexere Tätigkeit als üblich, da es letztlich um die Übertragung in eine andere Sprache und Kultur geht ${ }^{10}$. Daher muss sich der Übersetzungsstudent mit einer äußerst schwierigen Aufgabe auseinandersetzen für die er in den meisten Fällen noch unzulänglich ausgebildet ist. ${ }^{11}$ Der Dozent muss also klar feststellen, welche anfänglichen

anderer Leser- „Typus“ (einer der zum Beispiel nicht über die vorgesehene Kenntnisse verfügt) das Werk aktualisiert, so dass der Text an neuen Bedeutungen und Möglichkeiten gewinnt: „Nada más abierto que un texto cerrado." (1993:83).

${ }^{8}$ Siehe Acosta, L.A. (1989:139-140): "Las objeciones de G. Kaiser a los principios teóricos de Jauß tienen su fundamento y punto de partida en una concepción específica del texto (...) Kaiser ve las cualidades del mismo como una realidad inmanente y objetiva que, por esta misma razón, no puede variar con el transcurso del tiempo. (...) Por otra parte, objeta Kaiser, la teoría de la recepción, al basar sus análisis en la relación existente entre el lector y la obra, corre el peligro de menosvalorar y dejar un poco de lado la obra, convirtiéndola, de esta manera, en una realidad vacía de contenido, susceptible de ser llenada de un virtual significado".

${ }^{9}$ Genau dies ist das Ziel der Analyse Isers, der sich mit dem englischen Roman aus dem 17. bis zum $20 \mathrm{Jh}$. auseinandersetzte. S. diesbezüglich Iser, W., Der implizite Leser. Kommunikationsformen des Romans von Bunyan bis Beckett (1972).

${ }^{10}$ Enríquez (2007:15): "En primer lugar, el traductor desempeña a la vez un papel de receptor y de emisor. Se convierte, así, en el primer receptor del T.O. que debe traducir y en emisor del T.T. En opinión de Hurtado Albir $(2001,363)$ los procesos de comprensión y de expresión que el traductor debe acometer son particulares. De un lado, el traductor no suele pertenecer a la comunidad lingüístico-cultural en la que se inserta el T.O. y desde un principio tiene la intención de traducir el texto, por lo que su comprensión es "deliberada y más analítica" que la que realizaría cualquier receptor en una situación comunicativa monolingüe. De otro lado, el traductor ha de trabajar con un texto que ya ha sido emitido con anterioridad y en unas condiciones profesionales determinadas, por lo que su emisión también es, cuando menos, especial. En realidad, el traductor se puede considerar un receptor privilegiado y especializado".

${ }^{11} \mathrm{Vgl}$. Balbuena (2011:294): “(...) suele suceder que los alumnos que cursan esta materia no poseen una buena formación filológica en general y literaria en particular. Esto es aún más evidente en los casos en los que el alemán es una segunda lengua extranjera (Lengua C)". 
Fähigkeiten bei den Studenten verfügbar sind und welche Kompetenzen diese entwickeln sollten, um den Lernprozess angemessen zu leiten. Im Sinne von Umberto Eco ${ }^{12}$ müssen wir, ähnlich wie der Autor, den ModellLeser bestimmen, der im Rahmen der Lehrtätigkeit für Literaturübersetzungen ein Modell-Übersetzungsstudent ist. Gleichfalls müssen wir uns über die Fähigkeiten dieses Studenten bewusst sein, bevor wir inm einen bestimmten Text vorlegen.

Unser Ziel ist die Anwendung der Rezeptionsgrundlagen bei der Lehrtätigkeit, daher liegt es nahe, den Student der Literaturübersetzung als Leser zu kategorisieren und inn anderen, zeitgenössischen Lesertypen, Empfänger des gleichen Werks in vergleichbaren Kontexten, gegenüber zu stellen. Daher haben wir im Bereich deutsche Literatur und Sprache das derzeitige Profil des deutschen Schülers der Sekundarstufe II herangezogen. Der Roman Der Vorleser von Bernhard Schlink ist in den Lehrplänen verschiedener deutscher Bundesländer vorgesehen, unter anderem auch in Bayern ${ }^{13}$. Je nach Lehrplan wird der Roman von Schlink in der Klasse 10, 11 oder 12 behandelt (gleichzusetzen mit der Klasse 4 der Sekundarschule, der ersten und zweiten Oberstufenklasse in Spanien). Sein Stil ist direkt und nüchtern und der Roman beginnt im Jugendalter des IchErzählers, wobei in die komplexe Handlung persönliche und soziale Elemente (Justiz, Verbrechen der Nationalsozialisten) eingefügt werden. Auch die historische Thematik sorgt dafür, dass junge Leser erreicht werden und sich angesprochen fühlen. Neben der Lektüre bietet der Roman ebenfalls Raum zur Reflexion und Untersuchung der deutschen Vergangenheit, eine Thematik die nach deutschem Schulsystem Pflicht ist.

Nachfolgend wird der unterschiedliche Umgang mit dem Werk seitens der deutschen Oberschüler, die den Roman Der Vorleser im Deutschunterricht durchnehmen und der Übersetzungsstudenten der

\footnotetext{
${ }^{12}$ Auf der theoretischen Ebene hat die Literaturkritik bereits eine Reihe von Leserkonzepten geliefert, auf die zurückgegriffen wird, wenn Auswirkung oder Rezeption des Werks behandelt wird. Unter diesen Lesertypen befinden sich der sogenannte ideale Leser und der Leser der Epoche. Verschiedene Autoren haben gleichfalls spezifische Lesertypen benannt: Stanley Fish und sein Konzept des informierten Lesers, Michael Riffaterre und der Archileser (oder Lesergruppe "Informanten"), Erwin Wolff und der intendierte Leser oder der Leser, der im Geist des Autors gestaltet wurde. Auf gleicher Weise stellt Wolfgang Iser den impliziten Leser vor. Und Umberto Eco sieht den Modell-Leser als "un conjunto de condiciones de felicidad, establecidas textualmente, que deben satisfacerse para que el contenido potencial de un texto quede plenamente actualizado" (1993:89). Alle diese Konzepte können oder können nicht auf einen realen Leser zu einem bestimmten Zeitpunkt zutreffen, aber sie sollen lediglich als Grundstruktur der Literaturtheorie dienen.

${ }^{13}$ Siehe die empfohlenen Lektüren vom Staatsinstitut für Schulqualität und Bildungsforschung Bayern für die Klasse 12:

http://www.isb-gym8lehrplan.de/contentserv/3.1.neu/g8.de/data/media/26540/Lekt\%FCrevorschl\%E4ge\%20Jg.\%20 12.pdf
}

Hikma 13 (2014), 9-20 
Universität, die einen Teil desselben Werks im Hörsaal Literaturübersetzen bearbeiten, untersucht ${ }^{14}$. Hierfür sind die bedeutendsten Unterschiede, die uns beschäftigen, einerseits der Umgang mit dem Roman oder die Bearbeitung, die im Lehrraum vorgenommen wurde (unter Anbetracht der Lernziele) und andererseits die Vorkenntnisse oder Erfahrungen der Schüler/ Studenten mit dem Repertoire, im Sinne der Definition von Iser ${ }^{15}$.

Diese letzten beiden Punkte sind von starker Bedeutung beim Umgang mit dem Werk Der Vorleser im Hörsaal deutsches Literaturübersetzen.

3. Ansatz für eine angemessene Rezeption des Romans DeR Vorleser SEITENS DER LESERSCHAFT „ÜBERSETZUNGSSTUDENTEN“

Als ersten Aspekt bei der Planung der Lehrstunden muss bedacht werden, dass das gesamte Werk nicht gelesen wird, sondern dem Studenten lediglich ein Teil des Textes vorgelegt wird, ganz anders also als im Deutschunterricht der Oberstufe, wo die gesamte Lektüre wahrscheinlich Pflicht ist. Da die Lehrpläne für Literaturübersetzen, ein viermonatiger Kurs, außerdem eine weitere Vielzahl an Werken aus unterschiedlichen Epochen der deutschen Literatur vorsehen, stehen uns lediglich zwei oder drei Lehrstunden für Schlinks Werk zur Verfügung. Das konkrete Ziel ist, dass die Studenten einen sehr bekannten Roman der zeitgenössischen deutschen Literatur kennenlernen und dass sie Teile davon ins Spanische übersetzen. Die Zielsetzung in diesem Fach ist die Vermittlung von Grundkenntnissen deutscher Literatur, um so literarische Texte vom Deutschen ins Spanische übersetzen zu können: es handelt sich also um eine Einführung, keinesfalls um eine Spezialisierung.

Gemäß der Rezeptionstheorie aktualisiert der Leser den Text beim Leseprozess und erschafft somit die Bedeutung desselbigen. Die Übersetzungsstudenten haben den Nachteil, dass sie nicht den gesamten Roman lesen können und trotzdem wird gefordert, dass sie die vorliegenden Teile richtig übersetzen können, also wird ein angemessenes Verständnis des Originals erwartet. Um diesen ersten Nachteil auszugleichen ist es

14 Es muss erwähnt werden, dass der erste Unterschied zwischen den Lesern, Übersetzungsstudenten und deutschen Schülern der Oberschule, das Alter ist. Die deutschen Schüler sind zwischen 15 und 18 Jahre als, die spanischen Studenten aus dem Fachbereich Literaturübersetzen sind normalerweise zwischen 20 und 21, gegebenenfalls sind unter innen einige ältere Studenten. Austauschstudenten oder Studenten unterschiedlicher Nationalität werden nicht berücksichtigt. Die Übersetzungsstudenten sprechen Spanisch und lernen Deutsch als zweite Fremdsprache (C-Sprache), im Gegensatz dazu lesen die deutschen Schüler den Roman in ihrer Muttersprache.

${ }^{15}$ Iser (1975:267-268): ,Jeder literarische Text zieht in mehr oder minder großem Umfang und mit mehr oder minder deutlicher Markierung soziale, historische und zeitgenössische Normen sowie eine entsprechende Anspielungsvielfalt aus der literarischen Tradition in sich hinein. Sie bilden das dem Leser bekannten Repertoire des Textes." 
notwendig, dass das Werk den Studenten vorgestellt und ihre aktive Teilnahme gefördert wird, da andernfalls keine angemessene Rezeption erreicht werden kann und wahrscheinlich nicht die Bedeutung des Textes erkannt wird, was sich wiederum negativ auf ihre Übersetzungen niederschlägt.

Eine weitere Möglichkeit die Studenten mit einzubeziehen ist die Vorführung der englischsprachige Verfilmung The Reader (2008), die Verwendung von Arbeitsmitteln zur Zusammenarbeit und die gelenkte Reflexion.

\subsection{Die Intermedialität beim Roman „Der Vorleser“}

Die didaktische Verwendung von Filmmaterial ist ein bekanntes Mittel im Fachbereich Übersetzen ${ }^{16}$. In diesem Fall ergibt sich eine untypische Situation, da die Verfilmung vom Roman Der Vorleser eine Koproduktion zwischen den Vereinigten Staaten und Deutschland ist, daher auf Englisch gedreht wurde, auch wenn viele deutsche Schauspieler mitgewirkt haben. Bei der Vorführung kann es ein Vorteil sein, da unsere Studenten Deutsch als C- Sprache haben und Englisch meistens weitaus besser beherrschen.

Wir werden zwei der erwähnten Strategien von Balbuena verwenden: die Vorführung des Trailers im Lehrraum, um das Werk vorzustellen und die Projektion von ausgewählten Szenen, die zum besseren Kennenlernen der Figuren und Eigenschaften des Ausgangstextes führen.

Auf diese Weise schaffen wir einen intermedialen Bezug zwischen dem Der Vorleser und The Reader, indem wir zwei Unterkategorien von Rajewsky anwenden:

„I. Intermediality in the more narrow sense of medial transpositions (as for example film adaptations (...)

III. Intermediality in the narrow sense of intermedial refer-

ences (...). Hence, in the case of the film adaptation, the viewer "receives" the original literary text along with seeing the film, and specifically receives the former in its difference from or equivalence to the latter." (2005 : 51-52).

Die erste Unterkategorie (mediale Umsetzung) unterstützt uns dabei die Haupthandlung des Werks zu vermitteln, die Vorführung des Trailers

\footnotetext{
${ }^{16}$ Balbuena (2011:296): "Cuatro son las posibles actuaciones que proponemos como complemento a la traducción del texto en clase, empleando como recurso la proyección fílmica previa a la traducción de textos literarios. Son las siguientes: proyección de trailers en versión original; proyección en lengua alemana, dobladas al alemán o con subtítulos en alemán; proyección de filmes y comparación con el Filmbuch o guión; y, finalmente, proyección de películas que, sin ser adaptaciones cinematográficas de obras literarias, contribuyen al conocimiento de personajes y elementos propios del texto de partida".
} 
dient zur Einführung der Lehrstunde. Bezüglich der Unterkategorie III verwenden wir intermediale Referenzen des Films, um so das Verständnis für Schlüsselszenen vor der Übersetzung des entsprechenden Abschnitts zu schaffen und, worauf Balbuena hinweist, um das Wesen der Figuren darzustellen.

Es ist wichtig darauf hinzuweisen, dass das Ziel unserer Studie immer noch Schlinks Roman ist, weshalb die Verfilmung lediglich als „intermedialer Bezug" zu diesem Roman dient, also das Verständnis der ursprünglichen Elemente vervollständigt und nicht als eigenständiges Produkt betrachtet wird, ansonsten wäre es Objekt eines anderen Bereichs oder Kontextes.

\subsection{Die Hilfsmittel zur Zusammenarbeit}

Um die Beteiligung und Einbeziehung der Studenten bei der Erschaffung der Bedeutung des Werks zu unterstützen, schlagen wir vor, eine virtuelle Plattform zu verwenden, wie zum Beispiel Moodle oder ein Hilfsmittel zur Zusammenarbeit, in dem die Studenten die Inhalte zusammenfassen müssen, die dann bei der Bearbeitung des Romans richtungweisend sind. Typisch hierfür sind die Romanhandlungen, der Aufbau, die Wesenzüge der Hauptfiguren, die Themen die angesprochen werden, die lexische, morphologische und syntaktische Analyse der übersetzten Abschnitte und die Quellenverweise.

Deswegen wird erwartet, dass alle Studenten die Rezeption über das Werk belegen und dass die von innen erarbeiteten Abschnitte (jeder Absatz wird einem Studenten oder einer Gruppe zugewiesen) mit in die Endnote der zwei oder drei Lehrstunden zu diesem Roman einfließen.

Daher sollte es darauf hingewiesen werden, dass dieses Vorgehen im Allgemeinen von den Schülern der deutschen Oberstufe zum Umgang mit der Lektüre verwendet wird. Die neuen Technologien eröffnen uns tatsächlich Unmengen an Möglichkeiten was die Teilnahme und Verbreitung von Kenntnissen angeht ${ }^{17}$.

\subsection{Die gelenkte Reflexion}

Als letzten Aspekt der Bearbeitung des Romans Der Vorleser wird vor der objektiven Prüfung (die aus der Übersetzung eines nicht besprochenen Romanabschnitts besteht) die Reflexion durchgeführt und hierfür Fragebögen oder Zusatztexte seitens des Dozenten ausgeteilt, die die Erschaffung der Werksdeutung untermauern sollen. Ebenfalls sollten die ausgehändigten und besprochenen Zusatzmaterialien bestimmte Wissenslücken der Studenten bezüglich des Repertoires kompensieren, da

${ }^{17}$ Siehe Sánchez Vázquez (2005:103): "Ahora bien lo que el arte electrónico, computarizado o digital no puede aportar en el plano estético, o aporta limitadamente, sí puede ofrecerlo en el plano social al permitir compartir, extender o socializar la creación". 
unsere Studenten gar keine oder nicht ausreichende Kenntnisse darüber haben. Beispiele für diese Wissenslücken sind die Referenzen zu anderen literarischen Werken deutscher Tradition (in Der Vorleser: Emilia Galotti von Lessing, Kabale und Liebe von Schiller), die spezifische Konzepte, die dem spanischen Studenten nicht bekannt sind (Todesmarsch, Siebenbürgen) oder der historisch- soziale Kontext des Romans.

\section{SCHLUSSFOLGERUNGEN}

Der Übersetzer ist gleichzeitig Empfänger und Sender des Textes und daher verfügt er über ein anderes Profil als andere Lesetypen, die der Autor eines bestimmten Werks erfassen kann. Noch dazu kommt, dass die Studenten für deutsches Literaturübersetzen einem Ausbildungsprozess unterworfen sind, bei dem die Entwicklung von notwendigen Fähigkeiten gefordert wird. Im Allgemeinen sind weder die vorherigen Kenntnisse der Studenten, noch die Bearbeitung des Werks im Lehrraum typische Voraussetzungen für eine Lektüre oder professionelle Übersetzung: zeitliche Begrenzung, geringe Unterlagen und vorherige Kenntnisse sorgen dafür, dass das Werk in Abschnitten bearbeitet wird.

Aber da die Übersetzer (und die Übersetzungsstudenten) vor allem bewusste Leser der Werke, die sie bearbeiten, sein sollten, ist es notwendig, dass eine angemessene Rezeption stattfindet. Dieser Prozess betrifft nicht nur die Bedeutung des Werks, sondern auch die bestehenden formalen Strukturen des Originaltextes und somit letztendlich auch das Repertoire.

Daher ist es unumgänglich, dass erkannte Lücken oder fehlende Kompetenzen ausgeglichen werden, die die Rezeption des literarischen Werks betreffen. Oder anders ausgedrückt: das Lehrziel sollte die Entwicklung von notwendigen Kompetenzen seitens der Studenten sein, damit eine angebrachte Rezeption (und Übersetzung) der literarischen Texte durchgeführt werden kann. Dafür ist es notwendig, exakt die Kompetenzen und Fähigkeiten der Studenten vor Bearbeitung im Hörsaal zu ermitteln, was heißt: das Profil des Modell-Studenten für Literaturübersetzen zu kennen.

Die Rolle des Dozenten im Hörsaal Literaturübersetzen sollte begleitend sein oder als Erleichterung dienen: die Teilnahme unterstützen, Raum für Dialog und Zusammenarbeit geben, so dass der Student ausreichend Möglichkeiten zur Teilnahme und Kreativität hat. Wenn die Studenten die Werke als "reales“"Element erfahren können bei dem sie teilnehmen, begünstigt dies den ästhetischen Genuss und die Bearbeitung wird produktiver und zufriedenstellender sein.

REFERENCIAS BIBLIOGRÁFICAS

AcOSTA GÓmEZ, Luis A., El lector y la obra: teoría de la recepción literaria. Madrid: Gredos, 1989. 
BALBuena ToREZANO, María del Carmen, "Texto literario y texto fílmico: una propuesta para la enseñanza de la traducción literaria alemánespañol". En: Silvia Roiss, Carlos Fortea Gil, Belén Santana López (eds.), En las vertientes de la traducción e interpretación del/al alemán. Berlin: Frank \& Timme GmbH, 2011, pp. 293-302.

BENNET, Andrew (ed.), Readers and Reading. London: Longman Publishing, 1995.

BLÜHER, Karl Alfred, "La crítica literaria en Valéry y Borges". En: Revista Iberoamericana, Nr. 135-36, 1986, pp. 447-461. Disponible en línea en: http://revistaiberoamericana.pitt.edu/ojs/index.php/lberoamericana/article/downloa d/4214/4382 [Fecha de consulta: 20 de agosto de 2013].

Eco, Umberto, The Role of the Reader: Explorations in the Semiotics of Texts. Bloomington: Indiana University Press, 1979.

_- Lector in fabula: la cooperación interpretativa en el texto narrativo. Barcelona: Lumen, 1993.

ENRÍQUEZ ARANDA, María Mercedes, Recepción y traducción: síntesis y crítica de una relación interdisciplinaria. Málaga: Universidad de Málaga, 2007.

FISH, Stanley, „Literatur im Leser: Affektive Stilistik”. En: Rainer Warning (Hrsg.), Rezeptionsästhetik. Theorie und Praxis. München: Wilhelm Fink Verlag, 1975, pp. 196-227.

FREUND, Elizabeth, The return of the reader: reader-response criticism. London: Methuen, 1987.

HoLUB, Robert, Reception Theory: A critical introduction. London: Routledge, 1989.

ISER, Wolfgang, Der Implizite Leser - Kommunikationsformen des Romans von Bunyan bis Beckett. München: Wilhelm Fink Verlag, 1972.

__, El acto de leer: teoría del efecto estético. Madrid: Taurus, 1987.

—_, „Der Lesevorgang. Eine phänomenologische Perspektive“. En: Rainer Warning (Hrsg.), Rezeptionsästhetik. Theorie und Praxis. München: Wilhelm Fink Verlag, 1975, pp. 253-276.

- Prospecting: from reader response to literary anthropology. John Hopkins University Press, 1993.

JAUB, Hans Robert, Kleine Apologie der ästhetischen Erfahrung. Konstanz: Uvk Univers.-Vlg Konstanz, 1972.

__ Experiencia estética y hermenéutica literaria: ensayos en el campo de la experiencia estética. Madrid: Taurus, 1986.

__, Wege des Verstehens. München: Wilhelm Fink, 2001.

—_, "Literaturgeschichte als Provokation der Literaturwissenschaft". En: Rainer Warning (Hrsg.), Rezeptionsästhetik. Theorie und Praxis, München: Wilhelm Fink Verlag, 1975, pp.126-162. 
„Lektürevorschläge Jahrgangsstufe 12.“ Lehrplan für das Gymnasium in Bayern. Lehrplan des achtjährigen Gymnasiums. Staatsinstitut für Schulqualität und Bildungsforschung, 2012. Disponible en línea en: http://www.isb-gym8-

lehrplan.de/contentserv/3.1.neu/g8.de/data/media/26540/Lekt\%FCrev orschl\%E4ge\%20Jg.\%2012.pdf

[Fecha de consulta: 20 de agosto de 2013].

MAYORAL, José Antonio, Estética de la recepción. Madrid: Arco, 1987.

RAJEWSKY, Irina O., „Intermediality, Intertextuality, and Remediation: A Literary Perspective on Intermediality". En: Intermédialités: Histoire et Théorie des Arts, des Lettres et des Techniques, Nr. 6, 2005, pp. 4364.

SÁNCHEZ VÁZQUEZ, Adolfo, De la estética de la recepción a una estética de la participación. México: UNAM, 2005. 